\title{
Gain of Function Research: the Clairvoyant Lens on Pandemics
}

\author{
Sudhir BHANDARI ${ }^{1}$, Amitabh DUBE², Bhoopendra PATEL ${ }^{3}$, Amit TAK ${ }^{4}$, Minal KACHHAWA², \\ Jitendra Kumar GUPTA2 ${ }^{2}$ Kapil GUPTA², Shivankan KAKKAR ${ }^{5}$
}

\begin{abstract}
Pandemic influenza viruses have emerged three times in this century. It is important to examine the potential risk of novel microorganisms/viruses through the add-on research mechanism of Gain of Function Research (GoFR). This mechanism consists of the practice of serial passaging of microorganisms to increase their transmissibility, virulence, immunogenicity, and host tropism through the inclusive feature of selective pressure of culture medium. Although, the GoFR can be a double-edged sword that has the potential to give an insight and better appreciation of current and future pandemics with antecedent apprehension of initiating a pandemic, itself. Moreover, with its inherent potential to give a head start on a virus, GoFR has the potential to develop vaccines or therapeutics, before the virus emerges in its true virulent form. Likewise, the GoFR studies can be vital in research on antivirals and antimicrobial agents and can help inform the development of combination therapies. Passive immunotherapy, which often includes a combination of products, is particularly dependent on GoFR experiments for evaluating efficacy. GoFR if made use of meticulously and with caution could help Medical Sciences and Humankind tremendously..
\end{abstract}

Keywords: GoFR, influenza, microorganisms, pandemic, vaccines.

'Department of Medicine, SMS Medical College Hospital, Rajasthan, India

${ }^{2}$ Department of Physiology, SMS Medical College Hospital, Rajasthan, India

${ }^{3}$ Department of Physiology, AlIMS, Bilaspur, Himachal Pradesh, India

${ }^{4}$ National Centre for Disease, Bengaluru, Karnataka, India

${ }^{5}$ Department of Pharmacology, SMS Medical College Hospital,

Rajasthan, India

\section{Corresponding author.}

Shivankan KAKKAR, Department of Pharmacology, SMS Medical College Hospital, Jaipur-302004, Rajasthan, India.

E-mail: drshivankankakkar@gmail.com 


\section{INTRODUCTION}

Humankind has witnessed the trials and tribulations of pandemics, since times when humans changed the basic nature from hunter-gatherer to social/crowd-gatherer with a plethora of literature depicting the sordid picture of deaths and pyres burning everywhere. It seems that with changing times the causative microorganism also metamorphosis to evolve novel antigenic armamentarium to the immunological naive humans that subsequently leads to a hyper-immune response inclusive of cytokine storm, that seems to be the common denominator of all pandemics.

Pandemics seem to be besought and dazzled by the characteristic features of Influenza A Viruses, negative RNA viruses belonging to genus, Orthomyxoviridae, that possess the capability to interface (across species through the phenomenon of gene reassortment) and circulate in humans as annual epidemics, especially in the winters of temperate climes, with an ability to emerge as antigenically novel pandemic virus strains. The temperate zones of earth seem to be the breeding mechanistic areas of antigenic drift and shift for such labile and liable viruses that evolve into ghastly pandemic viruses. The pandemic influenza viruses have emerged three times in this century namely in the years 1918 („Spanish” influenza, H1N1), 1957 („Asian” influenza, H2N2), and 1968 („Hong Kong” influenza, H3N2) (Cox and Subbarao, 2000; Webby and Webster, 2003) $)^{1,2}$.

It would be worthwhile to note that to assess and explore such a singular phenomenon of the pandemic, efforts were initiated to fully examine the potential risk of such novel microorganisms/viruses through add-on research mechanism of Gain of Function Research (GoFR) [a mechanism that upholds the practice of serial passaging of microorganisms to increase their transmissibility, virulence, immunogenicity, and host tropism through the inclusive feature of selective pressure of culture medium] to appreciate the underlying potential pandemic mechanisms to be prepared well in advance for a pandemic, a phenomenon more akin to a soothsayer or a sorcerer. Subsequently, principles of Public Health/Community Medicine mandate that the infecting agent responsible for a pandemic be retrieved and preserved in maximal safety conditions to learn from lessons of history and give a probable insight into yet-to-come pandemics, its prevention, and therapeutics. Adhering to the dictates of Community Medicine,
Swedish-American Pathologist, Dr. Johan Hultin, the Indian Jones of Microbiology, in 1951 visited the Alaskan permafrost of a town called Brevig Mission, unearthed bodies of victims of Spanish Flu of 1918 (the origins of the virus has again been implicated to be due to genetic mutations within the geographic precincts of China) but could not isolate the virus. However, after almost 50 years, the Swedish-American Pathologist, Dr. Johan Hultin revisited Alaska and this time along with the team of virologists, Jeffrey Taubenberger and efforts to sequence the complete Spanish Flu 1918 virus were successful and in 2005 the whole of the virus was reconstructed, the benefits of which would be the assessment of antivirals/vaccines against a pandemic virus, support of epidemiological pandemic modeling, identification of determinants of virulence and transmission, surveillance, identification of novel viral proteins and study of the origin of the pandemic.

\section{CONCEPT OF GAIN OF FUNCTION RESEARCH (GOFR)}

Gain of Function Research (GoFR) is a categorical research mechanism wherein efforts are made to alter an organism or disease process to enhance and increase pathogenesis, transmissibility, or host range (type of hosts that a microorganism can infect) with the objectives of modeling and/or prognostication of emerging infectious diseases and to develop vaccines and therapeutics. The Gain of Function Research (GoFR) is a double-edged sword that has the potential to give an insight and better appreciation of current and future pandemics with antecedent apprehension of initiating a pandemic, itself. Moreover, with its inherent potential to give a head start on a virus, GoFR has the potential to develop vaccines or therapeutics, before the virus emerges in its true virulent form.

Gain of Function Research (GoFR) typically involves mutations that confer altered functionality of a protein or other molecule. These types of mutations have been utilized as incredible assets to comprehend the fundamental understanding of basic bacterial and viral biology and pathogen-host interactions. GoFR is salient to both the improved understanding of disease pathogenesis and the advancement of clinical countermeasures to irresistible infections, the debate over its safety and value is of direct relevance to medical and public health practitioners. GoFR usually results in the introduction of changes to biological agents that might 
increase their ability to infect a host and cause disease by enhancing their transmissibility or pathogenicity.

Genetic mutations have varying effects on the proteins that the specific gene is responsible for and is inclusive of no change in the protein, loss of function, or gain of function. Studies along these frontiers have been segregated as the setting of Dual Use Research of Concern (DURC) that can be reasonably envisioned to provide knowledge, information, products, or technologies, the applications of which could be distorted to pose a potentially significant hazard to public health and safety, crops and other plants, animal, environment, material and national security. Moreover, DURC could implicate conscious manipulation of pathogens as biological weapons or vice versa, wherein countermeasures are designed to evade such pathogens. DURC refers to double utilize research for which the consequences of malevolent use would be exceptionally severe (though practically any research might be considered "dual-use" broadly conceived - because almost any research, or simply about anything for that matter, are often used for a few malevolent purpose or other). Of particular concern with regards to life science research is that signs of progress in biotechnology may empower the advancement and utilization of a new generation of biological weapons that could be used for lethal mass destruction. However, despite such important potential benefits, GoFR can present dangers relating to biosecurity and biosafety. Some of the potential consequences of DURC that have been cited include the manipulation of pathogens for use as biological weapons and the development of mechanisms by which pathogens can evade countermeasures.

\section{BENEFITS AND RISKS OF GOFR}

The benefits of GoFR are varied and include the following:

- Scientific Knowledge: potentially unique information gained for better appreciation of pathogen/disease.

- Bio-Surveillance: enhancement of public health surveillance, agriculture and domestic animal surveillance, wildlife surveillance to improve outbreak detection/prediction and/or decision-making.

- Medical Countermeasures: potentially unique information facilitating the development of therapeutics, vaccines, and diagnostics.
- Informing Policy Decisions: regarding public health preparedness inclusive of countermeasure stockpiling, vaccine strain selection, resource mobilization.

- Economic Benefit: financial gains (from industrial productivity) and/or cost savings (from reduced health care expenses).

The risks of GoFR are manifold and the same needs to be treated with utmost caution and care. The following avenues need particular attention:

- Biosafety: dangers associated with laboratory accidents;

- Biosecurity: dangers associated with crime and terrorism if pathogens are not physically secure and/or if malevolent actors gain access to them;

- Proliferation: dangers that might grow proportionally with an increased rate of GOFR, potentially in different settings with varying biosafety standards;

- Information risk: if published studies facilitate malevolent action (e.g., by terrorists) or, possibly, breach of intellectual property;

- Agricultural: risks to agriculturally-relevant animals if enhanced pathogens arising from GOFR are accidentally or intentionally released into animal populations, and possible implications for human health;

- Economic risks: financial implications of (accidental or intentional) pathogen release or, possibly, opportunity costs; and

- Loss of public confidence: compromise of trust in the scientific enterprise that could result from (accidental or intentional) pathogen release.

\section{CLINICAL APPLICATIONS OF GOFR}

GoFR if made use of very meticulously and with caution could help Medical Sciences and Humankind tremendously, though its potential need to be addressed with caution.

- Animal Models

The development of novel prophylactic and therapeutic interventions invariably requires evaluation in animal models that, at least partially, recapitulate the disease in infected humans. Many emerging and remerging 
zoonotic diseases lack relevant animal models that closely recapitulate human disease (Safronetz et al, 2013) ${ }^{3}$. Subsequently, GoFR experiments are often needed to adapt virus isolates from humans to different, sometimes unnatural, mammalian hosts. Adaptation to some other or alternate new host inherently involves the alteration of pathogens through mutation. As the development of appropriate animal models can be a rate-limiting step in the evaluation of prophylactic and therapeutic interventions, GoFR modifications of viral strains can be an important tool toward accelerating the product development pipeline.

\section{- Vaccines}

Many live-attenuated vaccines, including some of the most successful vaccines ever developed, have been generated through GoFR techniques. From polio to smallpox to influenza, live attenuated vaccines elicit immunity against authentic epitopes on whole pathogens without causing disease. The live-attenuated measles vaccine was created by passaging the virus until mutations arose that altered virus tropism-a technique that could be considered, by current definitions, Gain of Function Research (GoFR) (Griffin and Pan, 2009) ${ }^{4}$. The introduction of GoF mutations attenuates the virus and provides an improved understanding of the mechanics of viral replication, thus potentially uncovering new strategies within the development of vaccines against emerging pathogens.

\section{- Therapeutic interventions}

The generation of escape mutants within the presence of an investigational agent is common practice for evaluating the role of antibiotics, antivirals, and other monoclonal antibodies. GoFR experiments with HPAIs (Highly Pathogenic Human Avian Influenza Viruses), for example, have identified specific mutations that can confer multidrug resistance (Baek et al, 2015; Marjuk et al, 2015) $)^{5,6}$. GoFR experiments are necessary for this context because naturally occurring resistant strains may not yet exist or the complex background of naturally occurring mutations may preclude identification of the amino acid residues that are critical to resistance

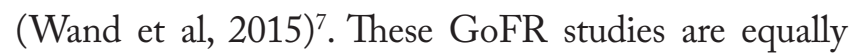
important in research on antivirals and antibiotics and can help inform the development of combination therapies. Passive immunotherapy, which often includes a combination of products, is particularly dependent on GoFR experiments for evaluating efficacy (de Jong et al, 2014; Keck et al, 2014; Caskey et al, 2015) ${ }^{8,9,10}$, as seen in the current Ebola outbreak that has prompted a robust program to evaluate combination monoclonal antibody therapies (Audet et al, 2014; Qiu et al 2014) $)^{11,12}$.

- Disease surveillance

In the past half-century, GoFR has contributed to an improved understanding of the epidemiology of emerging pathogens and has informed efforts to conduct surveillance for future outbreaks. GoFR experiments can be used to cross-reference traits found among circulating strains and help predict transmission patterns and pathogenicity (Davis et al, 2014) ${ }^{13}$. As the field of disease surveillance evolves to accommodate a growing repository of viral sequences, GoFR will also play an important role in assessing the public health significance of genotypic variation. The results of GoFR experimentation can also help inform decisions about countermeasure selection and stockpiling, particularly in the context of influenza surveillance programs (Schultz-Cherry et al, 2014) ${ }^{14}$. The improved understanding of how HPAIs evolve to transmit more efficiently has also factored into decisions about the creation of pre-pandemic vaccine stockpiles.

\section{- $\quad$ The COVID-19 pandemic and GoFR}

In late 2019, the argument was raised that the novel SARS-CoV-2 may have accidentally escaped from a high-containment laboratory in Wuhan, China (Metzl, $2020)^{15}$. It is a virus with all the hallmarks of a coronavirus from bat origin (the mammalian order known to be the largest reservoir of such type of viruses), but that displayed an enhanced ability to infect human cells through the singular Receptor-Binding Motif $(\mathrm{RBM})$ found in the virus's spike protein (that mediates the virus entry into host cells), an entity very similar to SARS-CoV, the implicated virus of 2003 SARS outbreak. Moreover, the novel SARS-CoV-2 contains a unique cleavage site for furine protease, an enzyme highly expressed in several human tissues like the brain, lung, pancreas, gut and kidney. The RBM and the furine protease cleavage site seem to be exclusively characteristic of the novel SARS-CoV-2.

As of now, the scientific consensus is that the virus emerged as a zoonosis whereby it jumped from an animal host, possibly bats or pangolins, to humans (Anderse 
et al, 2020) ${ }^{16}$, and arguments about a laboratory origin for SARS-CoV-2 are more akin to a conspiratorial theory than to a scientifically credible hypothesis. In the very unlikely event that SARS-Cov- 2 had emerged by accidental escape from a laboratory, however, that would be a great cause for concern because the Wuhan facility was state-of-the-art and presumably operating with maximal degree of care and caution. Regardless of how SARS-CoV-2 found its way into humans, what is certain is that the world's governments were caught off guard about the ways to respond. The ubiquitous ability of individuals to travel around the globe allowed the virus to spread rapidly before we knew what hit us, and even once we became aware, many countries reacted either too late or in arguably inappropriate ways, resulting in many thousands of avoidable deaths.

Taking all of this into consideration, following solutions can be posited for moving forward:

In the first place, there must be a straightforward audit of all GoFR facilities before their initiation, to guarantee that they are for sure resolving restoratively significant inquiries furthermore, that GoFR is the most ideal approach to acquire the appropriate responses.

These conversations should be public, also, choices cannot be made away from public scrutiny, as it appears was the case for decisions late last year by National Institute of Health (NIH), U.S.A. to allow new GoFR experiments on $\mathrm{H} 5 \mathrm{~N} 1$ to continue (Nglesby and Lipsitch, 2020 ${ }^{17}$. An absence of transparency only breeds distrust and suspicion and, if something untoward were to happen, might bring about a draconian reaction that could have far-reaching implications for the future of all research involving pathogens.

Second, giving biosafety the highest priority. All laboratories that perform experiments on highly pathogenic organisms should be required to adhere to a common set of standard protocols and procedures, including appropriate personal protective equipment (PPE). Moreover, for maintaining transparency, the results of regular inspections should be made publicly available. Laboratories must institute strict screening measures for his or her workers that often evaluate exposure, and protocols must be in situ to make sure that exposed workers don't transmit to others.

Third, there must be strong mitigation efforts in place, starting with detecting and preventing planned attacks. Similarly, there must be a strong surveillance program that watches zoonotic events. Such a program will require goodwill and cooperation with other countries and WHO. It is also essential that better ways to respond to any future events are evolved. For any transmissible disease, first and foremost, one would like to have a containment process that uses surveillance, testing, isolation, contact tracing to prevent spread, and the ability to test and produce therapeutics and vaccines.

\section{THE WAY FORWARD}

So, almost a decade after the great GoFR debate of 2011 to 2012, the COVID-19 pandemic has shown that the arguments from both sides have merit. The anti-GoFR camp's central argument that such experiments were too dangerous to conduct because humanity was too vulnerable to a pandemic proved correct in the sense that the world was unprepared for COVID19. On the other hand, the pro-GoFR camp's central argument that these experiments were necessary as information was needed on mechanisms of virulence and transmission also proved correct as humanity faced a new coronavirus with scant ability to spread and cause disease in humans. Going forward, humility is an essential precept to recognize that both sides have relevant intriguing issues and ways to obtain such information need to be explored to know while minimizing risks in the process.

Humans are always going to be faced with new infectious threats and in an interconnected world, the deadly pathogens with the right traits could acquire the ability to spread very rapidly. As a society, the following need to be explored and evolved:

- basic research to understand the biology of pandemic causing microorganisms and how they interact with their respective hosts;

- to research and find better diagnostics, therapeutics, and preventative measures to battle against pandemic like situations by applied research;

- better training for individuals working with/ on dangerous pathogens and guidelines for continuous monitoring of potential laboratory exposures;

- transparent review of proposed experiments for their long-term benefits and risks;

- public health bodies for monitoring and surveillance of potential new species jumps and outbreaks; and

- the ability to respond more rapidly and accurately to events if and when it occurs. 


\section{CONCLUSION}

A holistic approach like this will maximize and optimize benefits for Humankind and subsequently, with ongoing efforts the intricacies of the present COVID-19 pandemic could to a greater extent be envisaged with the development of an effective vaccine strategy and development of antiviral strategies, though antiviral therapeutics had to be customized along with ever-evolving clinical experience of the pandemic. It would be useful to add that strategies had been very carefully and meticulously planned, though Humankind could not match with the pace and wrath of the COVID-19 pandemic.

\section{References}

1. Cox NJ and Subbarao K. Global epidemiology of influenza virus: past and present. Annu Rev Med. 2000; 51: 407-21.

2. Webby RJ and Webster RG. Are we ready for pandemic influenza? Science. 2003; 302: 1519-22.

3. Safronetz D, Geisbert TW, Feldmann H. Animal models for highly pathogenic emerging viruses. Current opinion in Virology.2013; 3(2): 205-9.

4. Griffin DE, Pan CH. Measles: old vaccines, new vaccines. Curr Top Microbiol Immunol. 2009; 330: 191-21.

5. Baek $\mathrm{YH}$, Song M-S, Lee E-Y, et al. Profiling and characterization of influenza virus N1 strains potentially resistant to multiple neuraminidase inhibitors. J Virol. 2015; 89:287-99.

6. Marjuki H, Mishin VP, Chesnokov AP, et al. Neuraminidase mutations conferring resistance to oseltamivir in influenza $A(\mathrm{H} 7 \mathrm{N9})$ viruses. J Virol.2015; 89: 5419-26.

7. Wand ME, Bock LJ, Bonney LC, Sutton JM. Retention of virulence following adaptation to colistin in Acinetobacter baumannii reflects the mechanism of resistance. J Antimicrob Chemother. 2015.

8. De Jong YP, Dorner M, Mommersteeg MC, et al. Broadly neutralizing antibodies abrogate established hepatitis $C$ virus infection. Sci Transl Med. 2015; 6: 25-29.

9. Keck Z, Angus AGN, Wang W, et al. Non-random escape pathways from a broadly neutralizing human monoclonal antibody map to a highly conserved region on the hepatitis C virus E2 glycoprotein encompassing amino acids 412-423. PLoS Pathology. 2014; 10: e1004297.
Compliance with ethics requirements: The authors declare no conflict of interest regarding this article. The authors declare that all the procedures and experiments of this study respect the ethical standards in the Helsinki Declaration of 1975, as revised in 2008(5), as well as the national law. Informed consent was obtained from all the patients included in the study.

10. Caskey M, Klein F, Lorenzi JCC, et al. Viraemia suppressed in HIV1 -infected humans by broadly neutralizing antibody 3BNC117. Nature.2015.

11. Audet J,Wong G,Wang $\mathrm{H}$, et al. Molecular characterization of the monoclonal antibodies composing ZMAb: a protective cocktail against Ebola virus. Sci Rep. 2014;4: 68-81.

12. Qiu X, Wong G, Audet J, et al. Reversion of advanced Ebola virus disease in nonhuman primates with ZMapp. Nature. 2014; 514: 47-53.

13. Davis CT, Chen L-M, Pappas $C$, et al. Use of highly pathogenic avian influenza $\mathrm{A}(\mathrm{H} 5 \mathrm{~N} 1)$ gain-of-function studies for molecular-based surveillance and pandemic preparedness. Mbio. 2014; 5 .

14. Schultz-Cherry S, Webby RJ, Webster RG, et al. Influenza gain-offunction experiments: their role in vaccine virus recommendation and pandemic preparedness. Mbio. 2014; 5.

15. Metzl J. Origins of SARS-CoV-2.2020.

16. Andersen KG, Rambaut A, Lipkin WI, Holmes EC, Garry RF. The proximal origin of SARS-CoV-2. Nat Med.2020; 26:450 - 452.

17. Inglesby TV, Lipsitch M. Proposed changes to U.S. policy on potential pandemic pathogen oversight and implementation. mSphere.2020; 5:e00990-19. 